\title{
Mejora de la eficiencia hidráulica en una bomba hidráulica mediante modificación de la geometría de su rotor
}

\section{Improves of the hydraulic efficiency in a hydraulic bomb by means of modification of the geometry of their rotor}

\author{
Marcial De La Cruz L. ${ }^{(1)}$ \\ (1) Facultad de Ingeniería Mecánica Universidad Nacional del Centro del Perú \\ Email: mdelacruz@uncp.edu.pe
}

\section{RESUMEN}

El desarrollo de la investigación propone la modificación de la geometría a través del redimensionamiento de las partes constituyentes del rotor de las bombas hidráulicas para mejorar la eficiencia.

Se aplicó el método de investigación experimental debido a que se manipularon las variables de componentes del rotor nuevo a proponer. El tipo de investigación es el tecnológico y el nivel de investigación experimental. Se utilizó la técnica empírica para la recolección de datos. Se consideraron los datos de la bomba centrífuga con el rotor original y los datos de la bomba centrífuga con rotor nuevo, a través del modelado y la simulación con el software CatiaV5. Con estos datos se realizó la construcción de las curvas características para finalmente realizar las comparaciones y ver si mejora la eficiencia hidráulica. Se ha determinado que al modificar la geometría del rotor se mejora la eficiencia de la bomba hidráulica, el nuevo rotor desarrollado presenta una eficiencia de $8,6 \%$ más alto que el rotor original para el punto de mejor rendimiento; para ello, la bomba con rotor nuevo debe operar con caudales superiores a $5 \mathrm{~m}^{3} / \mathrm{h}$ y por debajo de $11,5 \mathrm{~m}^{3} / \mathrm{h}$. Además, el nuevo rotor demostró un efecto positivo en la curva de la potencia en comparación con el rotor original; la potencia consumida por la bomba operando con el nuevo rotor presentó una reducción de $0,42 k W$ en comparación con el rotor original en su respectivo punto de máxima eficiencia.

Palabras Clave: Eficiencia hidráulica - Bomba hidráulica - Geometría del rotor - Álabes - Caudal.

\begin{abstract}
The efficiency of the hydraulic bombs is not very good. The development of the investigation proposes the modification of the geometry through of the new measures of the constituent parts of the rotor to improve its hydraulic efficiency.

The method of experimental investigation was developed because the variables of components of the new rotor wer paleta curva e manipulated to propose. The investigation type is the technological one and the level of experimental investigation. The empiric technique was used for the gathering of data. They were considered the data of the centrifugal bomb with the original rotor and the data of the centrifugal bomb with new rotor through the modeling one and the simulation with the software Catia V5. With these data it was carried out the construction of the characteristic curves for finally to carry out the comparisons and to see if it improves the hydraulic efficiency.

It has been determined that modifying the geometry of the rotor improves the efficiency of the hydraulic bomb, the developed new rotor it presents an efficiency of 8,6 $\%$ higher that the original rotor for the point of better yield; for it the bomb with new rotor should operate with superior flows to $5 \mathrm{~m}^{3} / \mathrm{h}$ and below $11,5 \mathrm{~m}^{3} / \mathrm{h}$. The new rotor also demonstrated a positive effect in the curve of the power in comparison with the original rotor; the power consumed by the bomb operating with the new rotor presents a reduction of $0,42 \mathrm{~kW}$ in comparison with the original rotor in its respective point of maximum efficiency.
\end{abstract}

Keywords: Hydraulic efficiency - Hydraulic bomb Rotor geometry - Paddle - Plow. 


\section{INTRODUCCIÓN.}

Se ha determinado que modificando la geometría del rotor se mejora la eficiencia de la bomba hidráulica; el nuevo rotor desarrollado, presenta una eficiencia de $8,6 \%$ más alto que el rotor original, para el punto de mejor rendimiento; para ello, la bomba con rotor nuevo debe operar con caudales superiores a $5 \mathrm{~m}^{3} / \mathrm{h}$ y por debajo de $11,5 \mathrm{~m}^{3} / \mathrm{h}$. Con esto se ha cumplido con el objetivo principal, el de mejorar la eficiencia hidráulica de una bomba centrífuga.

Para una mejor comprensión de este estudio, se ha dividido en cuatro partes principales: el marco teórico, los materiales y métodos, los resultados y la discusión. En el marco teórico se ha considerado los conceptos sobre bombas centrífugas, clasificación de bombas y las bombas rotodinámicas; así mismo, se ha desarrollado la teoría de los principios de trabajo de la bomba centrífuga. En materiales y métodos se ha considerado el diseño de la investigación, la determinación del número de álabes, las condiciones de salida del rotor y el esquema experimental. En los resultados se presentan los coeficientes empíricos en el dimensionamiento del nuevo rotor, los parámetros de diseño y el modelado del rotor nuevo. En la última parte, que es la discusión se ha realizado la comparación de las características del rotor original con el rotor nuevo, fundamentalmente en lo que respecta a su eficiencia hidráulica. Finalmente se presentan las conclusiones del trabajo de investigación.

\section{MATERIALES Y MÉTODOS}

\section{Método tipo y nivel de investigación}

Se desarrolló el método de investigación experimental debido a que se manipularon las variables de componentes del rotor nuevo a proponer. El tipo de investigación es el tecnológico y el nivel de investigación experimental.

Se utilizó la técnica empírica para la recolección de datos. Se consideraron los datos de la bomba centrífuga con el rotor original y los datos de la bomba centrífuga con rotor nuevo. Con estos datos, se realizó la construcción de las curvas características, para finalmente realizar las comparaciones y ver si se mejora la eficiencia hidráulica. Se empezó con las características preliminares del rotor original de la bomba de ensayo en estudio, las cuales fueron:

- Rotor abierto;

- Rotor radial con un $n q=24$

- De una sola etapa;

- Rotor con 5 cuchillas o álabes

Para el análisis de la superficie de los álabes, se puede verificar que el rotor está fabricado por el proceso de fundición en arena. Se observa que en las entradas el material se aplica al método de desbarbado manual debido a las irregularidades detectadas en las paredes del rotor.

En los puntos donde los ajustes son más finos, es necesario (parte superior de los álabes y fijación del eje) el proceso de fundición y es complementado con el proceso de mecanizado.

\section{Principios técnicos de diseño del impulsor}

El método geométrico de pre-dimensionamiento para rotores es un proceso empírico e iterativo. Para renunciar a un modelo más completo en favor de la simplicidad, se impone la necesidad de coeficientes y constantes empíricas para establecer los resultados de los cálculos y mejores condiciones operativas para la bomba hidráulica.

Condiciones de aplicación del método geométrico:

$$
10<n q<30
$$

Para rotaciones específicas inferiores a 10 , la relación entre los diámetros del borde de presión y de succión excede 2,5. En esta situación los canales de los álabes serían muy largos y estrechos, y con ello, las pérdidas por fricción estrangularían al rotor.

A partir de la ecuación de Euler para una máquina hidráulica se puede determinar el trabajo específico teórico.

$$
Y_{\text {th }}=P / m i=c_{u 2} u_{2}-c_{u 1} u_{1}
$$

\section{a. Determinación de las condiciones de entra- da del rotor}

Figura 1. Rotor en corte longitudinal y sus dimensiones básicas

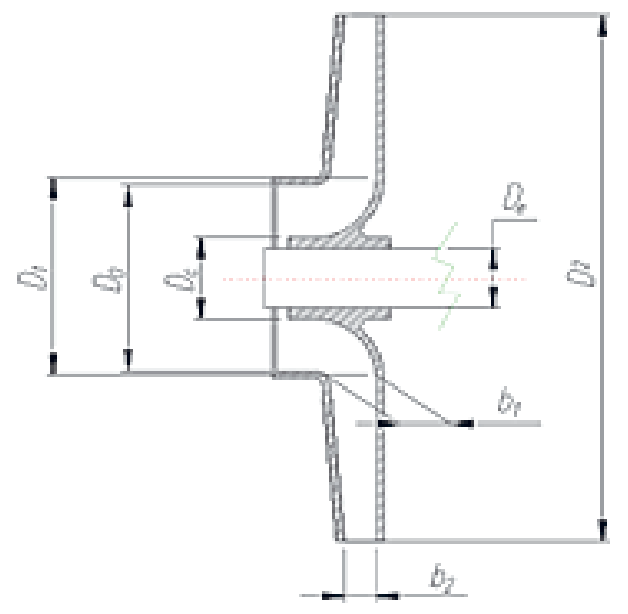

\section{b. Determinación del diámetro del cubo Dc}

El diámetro del cubo debe tener el diámetro más pequeño posible para que así se minimice la restricción del flujo en la entrada del rotor. Se sugiere una aproximación para el cálculo de acuerdo con la siguiente ecuación.

$$
C c=D_{e}(J)
$$

Siendo:

$$
1,35<J<1,5
$$

\section{c. Cálculo del caudal máximo Qm}

Para efecto del dimensionamiento del rotor, se debe considerar las pérdidas volumétricas del líquido resultante de las holguras entre el rotor y la carcasa. Estas pérdidas representan alrededor del 5\% del flujo de una 
bomba. Por lo tanto, el caudal máximo de la bomba se puede calcular con la ecuación:

$$
Q m=Q(1,05)
$$

\section{d. Cálculo de la potencia útil aplicada}

Se puede definir la potencia útil aplicada de una bomba a través de la ecuación:

$$
P_{u}=\left(\rho g Q_{m} H\right) / \eta_{u}
$$

Donde:

$\eta \_u$ : Es el rendimiento útil y debe ser obtenido por correlación con máquinas similares.

Para las pequeñas bombas se usa un rendimiento útil de 70\%.

\section{e. Cálculo del momento torsor}

El momento torsor al cual el eje del motor se somete puede ser determinado utilizando la ecuación:

$$
M=P_{u} / \omega
$$

\section{f. Cálculo del diámetro del eje $D_{e}$}

El diámetro del eje se puede obtener como una función del momento torsor que el eje debe transmitir y de la solicitud de torsión admisible.

$$
D_{e}=\left[16 M\left(\pi \tau_{t}\right)^{\wedge}(-1)\right]^{\wedge}(1 / 3)
$$

La ecuación es una simplificación, pues no se considera las tensiones de flexión del eje. Para máquinas de varias etapas, cuya distancia entre los cojinetes de la bomba es mayor, no es aplicable.

g. Cálculo de la velocidad de entrada del rotor $C_{0}$ Se admite que en el punto óptimo el flujo dentro del rotor no tiene rotación (entrada irrotacional). Así se forma el triángulo de velocidades a la entrada del rotor, el cual será un triángulo rectángulo; por lo tanto; $\alpha_{0}=90^{\circ}$ como se muestra en figura siguiente.

Figura 2. Triángulo de velocidades en la entrada del rotor (entrada irrotacional)

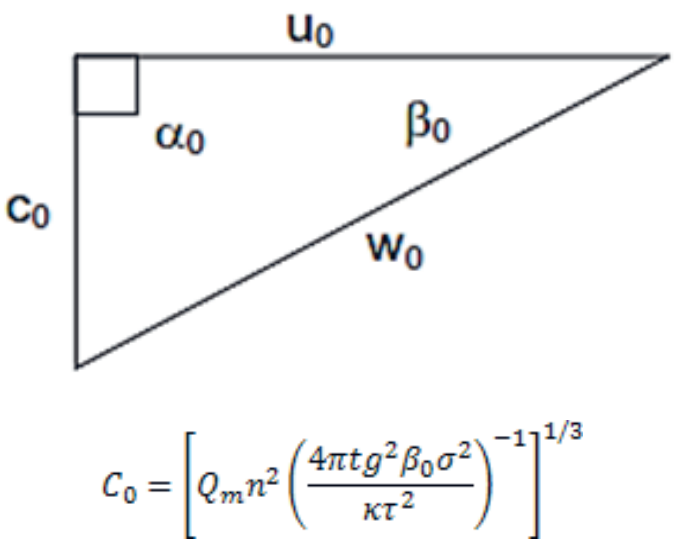

Donde:

$\sigma$ y $\tau$ : coeficientes función del tipo de alabes cuyo rango recomendado es:

$0,9<\sigma<1,1$

$1,05<\tau<1,1$

$k$ : es el coeficiente función del tipo de soporte del rotor (equilibrio, bi-soporte)

$0,75<k<0,85$

Se sugiere un valor de $\beta_{0}$ de aproximadamente 17 a $22^{\circ}$.

Además se proponen valores entre 12 a $22^{\circ}$ aumentando también la condición de que $\beta_{1}$ es mayor que $\beta_{0}$.

\section{h. Cálculo del diámetro de entrada del rotor $D_{0}$}

El diámetro de entrada del rotor está dado por la suma del diámetro del cubo con el diámetro externo de la corona circular del paso del fluido de acuerdo con la ecuación:

$$
D_{0}=\left[\left(\frac{Q_{m}}{c_{0}}+\frac{\pi D_{c}^{2}}{4}\right) \frac{4}{\pi}\right]^{0,5}
$$

\section{i. Cálculo de diámetro de entrada del rotor $D_{1}$}

El diámetro $D_{1}$ en el que se encuentran las entradas de los álabes debe ser mayor que el diámetro de entrada del rotor, porque en este caso, se observa una mejora en la capacidad de succión y una mayor estabilidad de la curva característica de la bomba.

$$
\text { Donde } \alpha<1 \quad D_{1}=\alpha D_{0}
$$

\section{j. Cálculo de la longitud de la entrada en el rotor $b_{1}$}

A partir de la aplicación de la ecuación de la continuidad se puede obtener la longitud del álabe en el lado de succión $b_{1}$.

$$
b_{1}=\frac{Q_{m}}{\pi D_{1} c_{0}}
$$

\section{k. Cálculo del ángulo de entrada en los álabes $\beta_{1}$}

El ángulo de entrada en los álabes se puede calcular a partir del análisis del triángulo de velocidades en la entrada del álabe (ver figura).

Figura 3. Triángulo de velocidades en la cara de succión

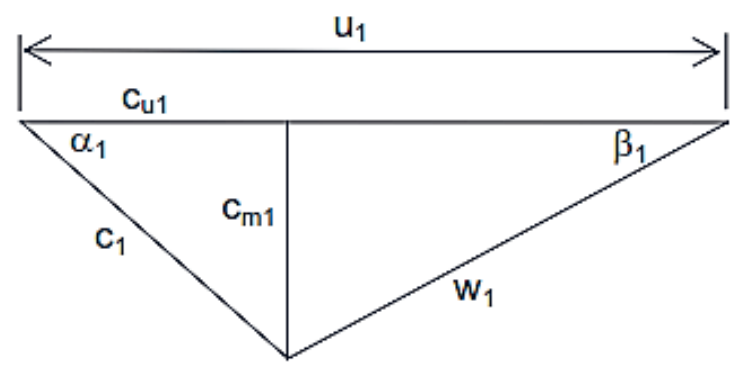

A partir del diámetro $D_{1}$ calculado anteriormente, se puede determinar la velocidad $u_{1}$

El factor de estrechamiento $\xi$ depende del paso entre los ála- 
bes $t_{1}$ y el espesor $\mathrm{s}$. El espesor del álabe, a su vez debe ser tan pequeño como el proceso de fabricación del rotor lo permita.

Este factor debe ser estimado por ahora, pendiente de verificación después de determinar el número de álabes z.

El ángulo $\beta_{1}$ debe estar comprendido dentro del rango $16-18^{\circ}$.

\section{Diseño de la investigación}

Se utilizó el diseño de experimentos unifactorial, donde la variable de entrada fue la bomba hidráulica con sus dos tratamientos con rotor original y con nuevo rotor y la variable de salida que fue la eficiencia hidráulica.

\section{Esquema experimental}

En esta parte se presenta todo el esquema experimental utilizado en el desarrollo de los experimentos.

El software Catia V5 se utilizó para comparar e investigar los efectos de las modificaciones introducidas en el rotor de la bomba analizada, como se muestra en la Figura 4.

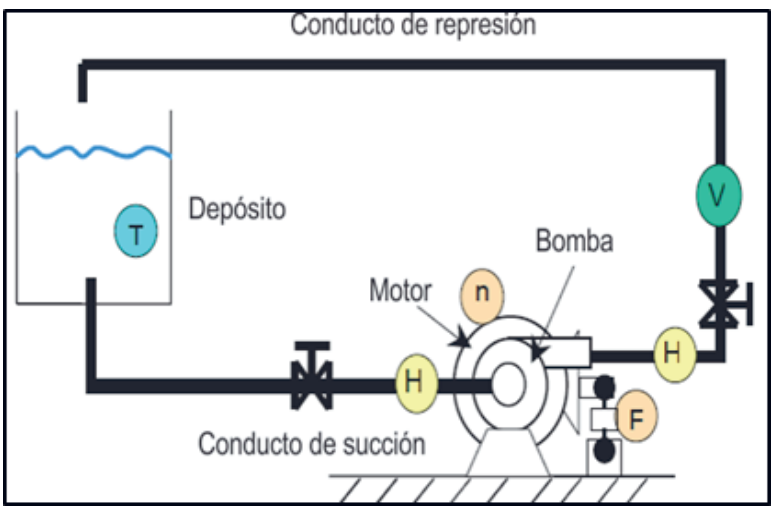

Figura 4. Esquema del experimento. T: Deposito; H: Puntos de toma de presión; F: Medición de la fuerza de reacción; $\mathrm{n}$ : Rotación del motor; V: Caudalímetro de presión diferencial

\section{RESULTADOS}

Para el pre dimensionado se utilizaron los coeficientes empíricos de las siguiente tablas:

Tabla $\mathbf{N}^{\circ}$ 01. Coeficiente empíricos utilizados en el dimensionamiento de un nuevo rotor

\begin{tabular}{lc}
\hline Coeficiente & 7 \\
Coeficiente & 1 \\
Coeficiente & 0,98 \\
Coeficiente & 1,05 \\
Coeficiente & 0,85 \\
\hline \hline
\end{tabular}

Tabla $\mathbf{N}^{\circ}$ 02. Característica constructivas del nuevo rotor

\begin{tabular}{lcc}
\hline \multicolumn{1}{c}{ Característica } & Valor & Unidad \\
\hline Grosor del álabe & 0,00335 & $\mathrm{~m}$ \\
Rendimiento total & $70 \%$ & $\%$ \\
Rendimiento hidráulico & $85 \%$ & $\%$
\end{tabular}

\begin{tabular}{lcc} 
Beta cero & 13 & $\circ$ \\
(máximo) & 0,127 & $\mathrm{~m}$ \\
(mínimo) & 0,097 & $\mathrm{~m}$ \\
(admitido) & 0,1246 & $\mathrm{~m}$ \\
\hline
\end{tabular}

Tabla $\mathrm{N}^{\circ}$ 03. Parámetros de diseño calcular para el nuevo rotor

\begin{tabular}{lcc} 
& Parámetros & Unidad \\
\hline Trabajo específico & 245,25 & \\
Rotación específica & 24 & -- \\
Diámetro de entrada & 0,059 & \\
Diámetro de entrada & 0,061 & \\
Velocidad tangencial & 11,1 & \\
Longitud del álabe & 18,06 & --- \\
Numero de álabes & 6 & $\circ$ \\
Ángulo & 18 & \\
Ángulo & 13,5 & \\
Diámetro de salida & 0,124 \\
Longitud de salida & 0,005 & \\
\hline
\end{tabular}

\section{Modelado del rotor nuevo.}

Para el modelado y la simulación para la obtención de los datos con el nuevo rotor se utilizó el software CatiaV5.

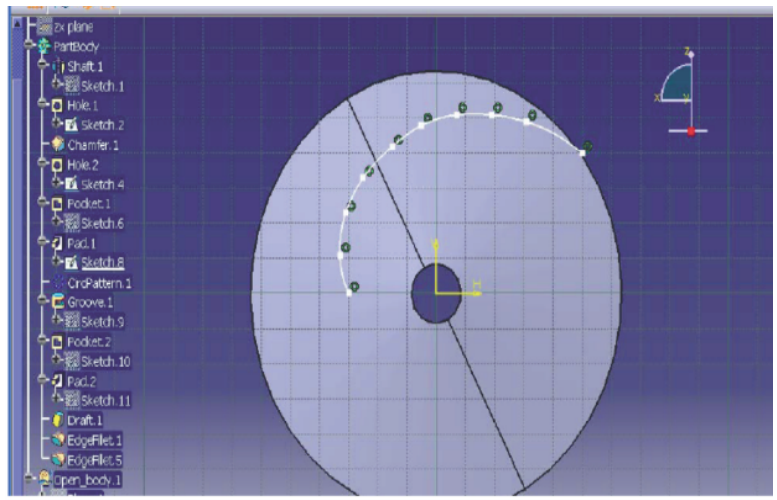

Figura 5. Detalle de la creación del perfil de los álabes del rotor

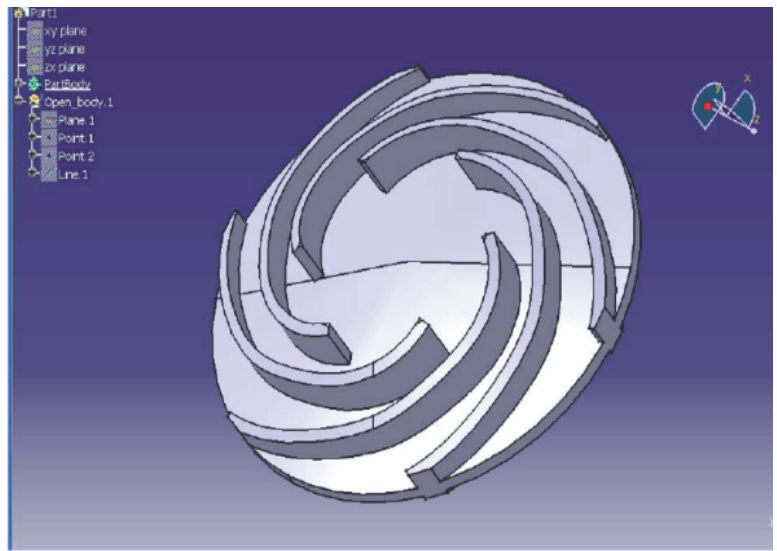

Figura 6. Rotor modelado a partir de sus dimensiones básicas 


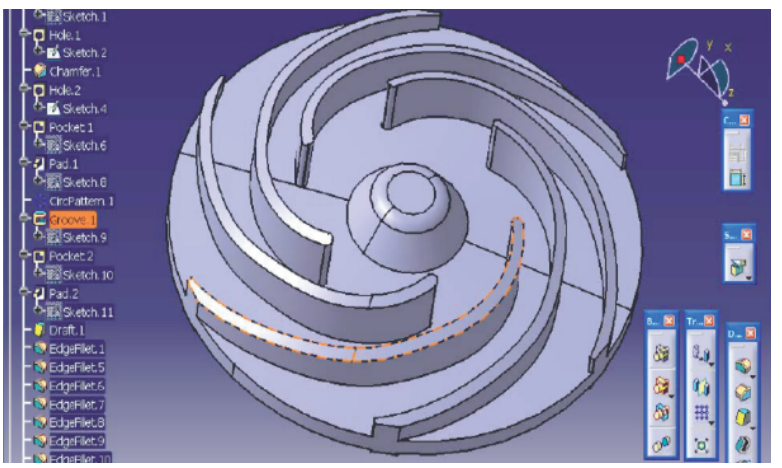

Figura 7. Rotor con cubo y radio en la entrada de los álabes

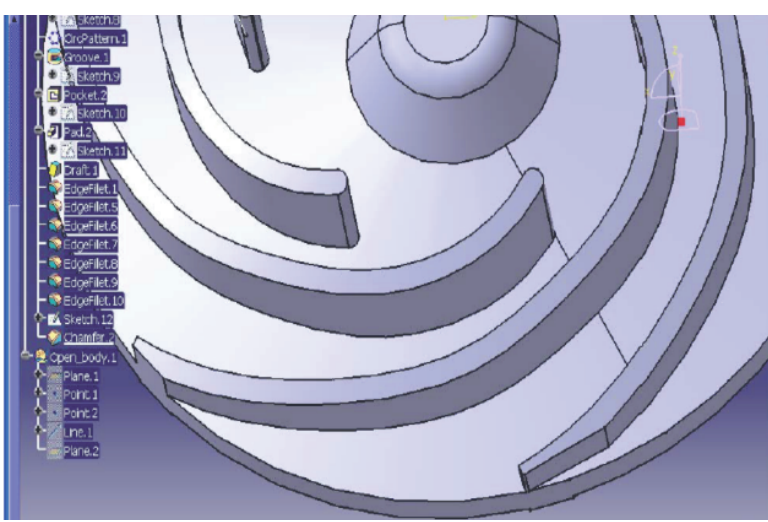

Figura 8. Detalle del refinamiento de los álabes

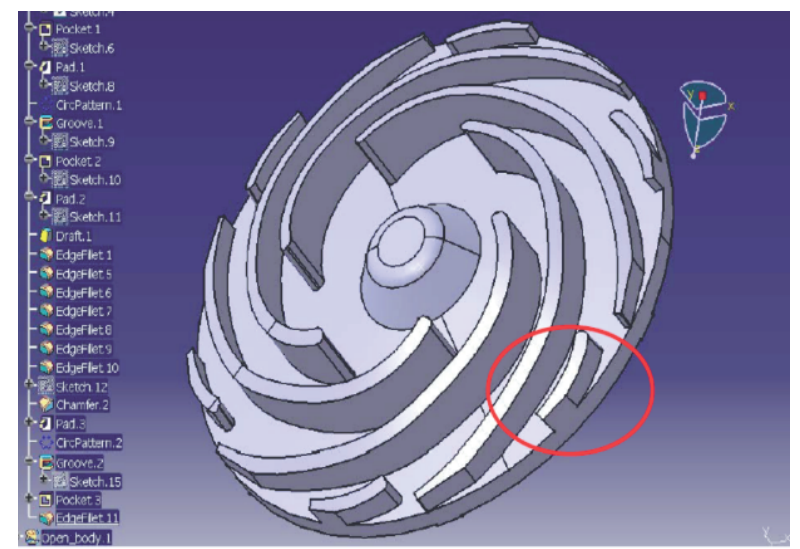

Figura 9. Rotor modelado con álabes direccionadores

\section{DISCUSIÓN}

Tabla $\mathbf{N}^{\circ}$ 04. Características del rotor original y el nuevo.

\begin{tabular}{lcc}
\hline \multicolumn{1}{c}{ Características } & Original & Nuevo \\
\hline Número de álabes & 5 & \\
Rotación específica & 24 & 24 \\
Diámetro de entrada & $38 \mathrm{~mm}$ & $59 \mathrm{~mm}$ \\
Diámetro de entrada & $42 \mathrm{~mm}$ & $61 \mathrm{~mm}$ \\
Diámetro de salida & $124 \mathrm{~mm}$ & $124 \mathrm{~mm}$ \\
Longitud del álabe & 3,8 a $4,8 \mathrm{~mm}$ & $3,25 \mathrm{~mm}$ \\
Álabes direccionadores & no & $\mathrm{si}$ \\
\hline
\end{tabular}

\section{Curva característica de cada rotor}

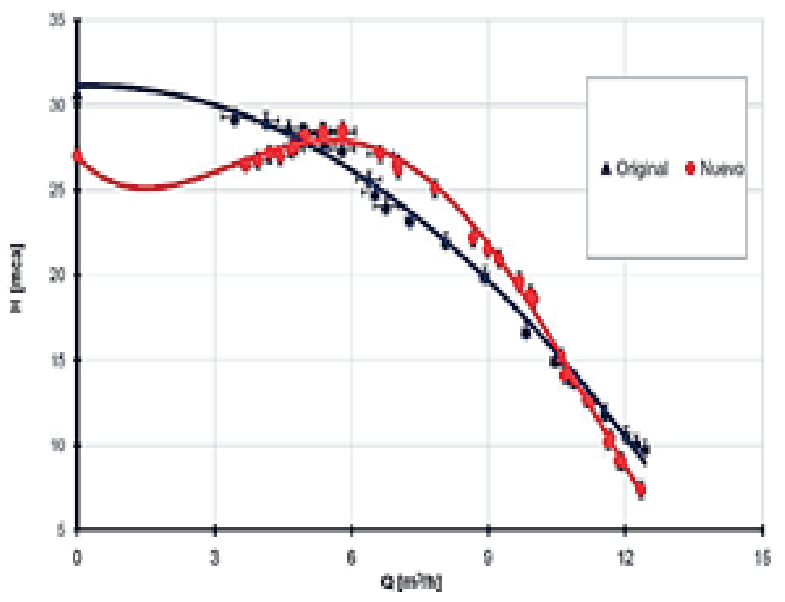

Figura 10. $f(H)=Q$ - Curvas características de los rotores

La curva característica para el rotor original de la bomba, mostró un comportamiento típico de una bomba centrífuga de velocidad especifica intermedia, en la cual la altura de elevación disminuye suave y regularmente a medida que el caudal aumenta.

Por otro lado, la curva característica de la bomba de prueba operando con el nuevo rotor presentó un comportamiento muy diferente. Entre el punto deshutt down (caudal cero) y el caudal de $6 \mathrm{~m}^{3} / \mathrm{h}$ el rotor demostró ser inestable y puede ser posible imponer la misma carga para diferentes caudales. También, dentro de este rango de operación, durante los ensayos se verificó una fuerte vibración en la bomba.

Este comportamiento no se mantiene para caudales superiores a $6 \mathrm{~m}^{3} / \mathrm{h}$ donde el nuevo rotor mostró una disminución regular de carga en función del aumento del caudal y también no se advirtió ningún tipo de vibración.

\section{Curva de la eficiencia}

En la Figura 11, se presentan las curvas de la eficiencia de la operación en función al caudal para cada modelo del rotor.

El análisis de las curvas de la eficiencia de ambos rotores muestra un comportamiento similar. Los puntos de máxima eficiencia de los rotores nuevo y original se obtuvieron en $7,82 \mathrm{~m}^{3} / \mathrm{h}$ y $8,91 \mathrm{~m}^{3} / \mathrm{h}$.

El rotor desarrollado presenta una eficiencia de 8,6\% más alto que el rotor original para el punto de mejor rendimiento. Por otra parte, hubo una disminución en la eficiencia para caudales por debajo de 5 y superiores a $11,5 \mathrm{~m}^{3} / \mathrm{h}$.

Esta mejora en la eficiencia operativa alcanzada por el nuevo rotor puede haber sido influenciada por el proceso de mecanizado, ya que esto proporciona un mejor acabado superficial y homogeneidad en la posición y el grosor de los álabes. 


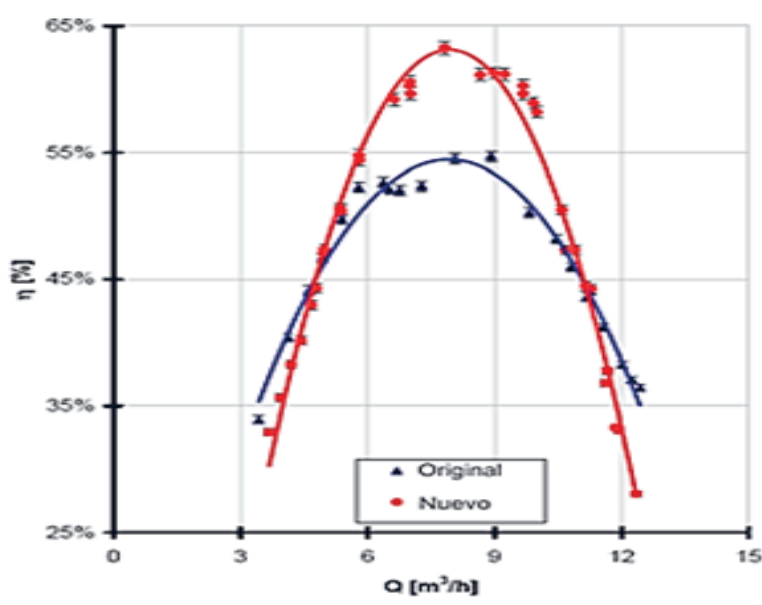

Figura 11. $\eta_{g}=f(Q)$ - Curva de la eficiencia para cada rotor

\section{Curva de la potencia}

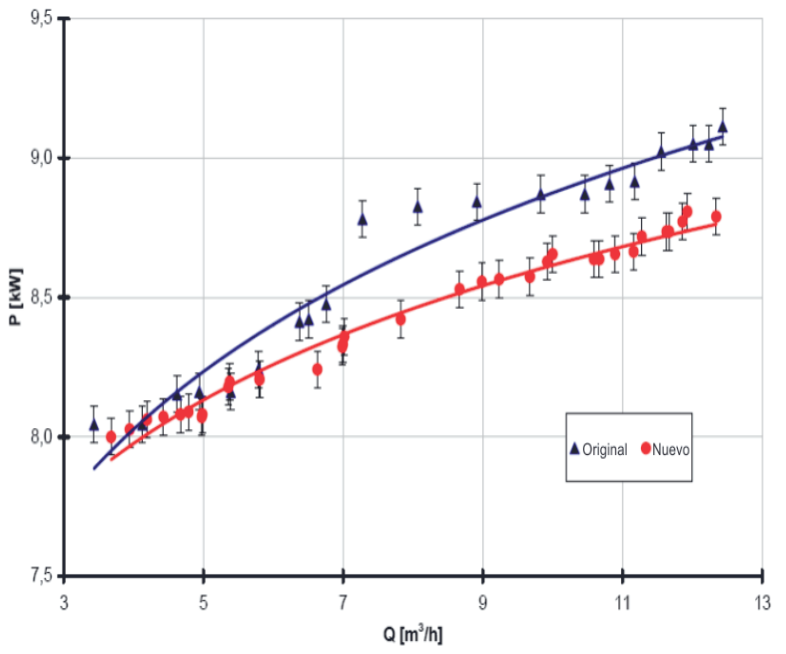

Figura 12. $P=f(Q)$ - Curva de la potencia para cada rotor

El nuevo rotor demostró un efecto positivo en la curva de la potencia en comparación con el rotor original. La potencia consumida por la bomba, operando con el nuevo rotor, presentó una reducción de $0,42 \mathrm{~kW}$ en comparación con el rotor original en su respectivo punto de máxima eficiencia.

\section{CONCLUSIONES}

- La curva característica para el rotor original de la bomba, mostró un comportamiento típico de una bomba centrífuga de velocidad especifica intermedia, en la cual la altura de elevación disminuye suave y regularmente a medida que el caudal aumenta; mientras que con el rotor nuevo, presentó un comportamiento muy diferente, entre el caudal cero y el caudal de 6 $\mathrm{m}^{3} / \mathrm{h}$. El rotor demostró ser inestable con una fuerte vibración en la bomba. Este comportamiento no se mantiene para caudales superiores a $6 \mathrm{~m}^{3} / \mathrm{h}$.

- El análisis de las curvas de la eficiencia de ambos rotores muestra un comportamiento similar. Los puntos de máxima eficiencia de los rotores nuevo y original se obtuvieron en $7,82 \mathrm{~m}^{3} / \mathrm{h}$ y $8,91 \mathrm{~m}^{3} / \mathrm{h}$. El rotor desarrollado presenta una eficiencia de $8,6 \%$ más alto que el rotor original para el punto de mejor rendimiento.

- El nuevo rotor demostró un efecto positivo en la curva de la potencia en comparación con el rotor original. La potencia consumida por la bomba operando con el nuevo rotor presentó una reducción de $0,42 \mathrm{~kW}$ en comparación con el rotor original en su respectivo punto de máxima eficiencia.

\section{REFERENCIAS BIBLIOGRÁFICAS}

Ariel, R. (2010). Metodología de diseño preliminar para pequeñas turbinas de reacción. Mexico: Mc Graw Hill.

Avella, J. (2013). Ahorro de energía en sistemas de bombas centrífugas. Sevilla España: International Tomson Editores.

Ayala, J. (2006). Optimización por diseños experimentales (Segunda reimpresión ed.). Lima Perú: Publicación de Concytec.

Caballero, A. (2012). Metodología de la investigación científica diseños con hipótesis explicativas. Lima Perú: Editorial Udegraf S.A.

González, J.; Fernández, J.; Argüelles, K. y Guerras, F. (2010). Análisis de tensiones determinísticas en una bomba centrífuga de doble aspiración. USA: Mc Graw Hill.

Gutierrez, H. (2008). Análisis y diseño de experimentos (Segunda edición ed.). México: Editorial Mc Graw Hill.

Hernández, R.; Fernández, C. \& Baptista, M. (2010). Metodología de la investigación. México: Mc Graw Hill Interamericana Editores S.A. De C.V.

J., G., J., F., \& E., B. (2011). Simulación numérica de los efectos dinámicos debido a la interacción de álabes-voluta en bombas centrífugas. México: Mc Graw Hill.

Montgomery, D. (2007). Diseño y análisis de experimentos (Tercera edición ed.). México: Editorial Limusa S.A. de C.V. Grupo Noriega Editores.

Pfleiderer, C. (2012). Bombas centrífugas y turbocompresores. México: Mc Graw Hill Interamericana Editores.

Sterling Group. (2012). Principios básicos para el diseño de instalaciones de bombas centrífugas. España: Sterling Group.

Velasquez, A. (2006). Metodología de la investigación científica. Lima Perú: Editorial San Marcos.

Wilo pumping. (2011). Principios fundamentales de la tecnología de las bombas centrífugas. Monterrey: Wilo pumping. 